\title{
Los cuerpos de Foucault: una genealogía de los estudios foucaultianos en el campo de académico de la Educación Física en Brasil y en la Argentina
}

CDD. 20.ed. 796.017

http://dx.doi.org/10.1590/1807-55092014000300453
Santiago PICH*

Norma Beatriz RODRIGUEZ**

\section{Resumen}

Este artículo tiene como objetivo analizar las investigaciones desarrolladas en el campo académico de la Educación Física en Argentina y Brasil basadas en la obra de Michel Foucault. Delimitaremos nuestro análisis del caso brasileño a tres pesquisadores que son, Homero Luis Alves de Lima, Alex Branco Fraga y Luiz Carlos Rigo y en el caso argentino, Ángela Aisenstein, Ricardo Crisorio y Pablo Ariel Scharagrodsky. Mostramos cómo esos trabajos están mayoritariamente referenciados en el segundo "dominio foucaultiano" y que son muy importantes para la denuncia de condiciones como la disciplinarización de los cuerpos y el control biopolítico de la población, sin embargo, el "tercer dominio" foucaultiano no es referenciado. Entendemos que ese dominio sería muy útil para la construcción de una propuesta pedagógica para la Educación Física, más allá del mero criticismo.

Palavras-chave: Michel Foucault; Campo académico; Argentina y Brasil; Pedagogía de la educación física.

\section{Introducción}

El siglo XX puede ser considerado como el "siglo del giro corporal” en las ciencias sociales, apropiándonos de las palabras de Bryan Turner. El cuerpo pasa a ganar estatuto ontológico en la constitución del ser humano y, en ese movimiento, algunos autores se destacan; siendo el filósofo francés Michel Foucault uno de sus protagonistas, juntamente con el filósofo Maurice Merleau-Ponty y los sociólogos Norbert Elias y Pierre Bourdieu, además de los antropológos Bryan Turner y David Le Breton, por citar a algunos de los más destacados pensadores. En ese movimiento se ha constituido un "campo de estudios sobre el cuerpo" de carácter multi- e interdisciplinario que comparte un objeto y que, sin embargo agrega una gran diversidad de posicionamientos teórico-metodológicos, que no serán aquí objeto de discusión.

Ese movimiento en el interior de las humanidades y de las ciencias sociales está siendo una referencia importante para los emprendimientos académicos desarrollados desde la Educación Física en el Conosur desde los años 80 del siglo XX. En este sentido, Brasil ha sido el país de América del Sur que en primer lugar se hizo eco de ese cuerpo teórico, y posteriormente en Argentina también se ha conformado un campo académico que se nutre de esas referencias. Particularmente en Brasil, el "movimiento renovador"a de la Educación Física brasileña ${ }^{1}$, entendía a la crisis de identidad epistemológica ${ }^{2}$, como necesaria para una refundación del campo. Debemos destacar, sin, embargo, que los integrantes de ese movimiento, se nutren de gran diversidad de referenciales teórico-metodológicos, que varían desde el marxismo ortodoxo, la escuela de Frankfurt, las teorías bourdeana y elisiana y la obra foucaultiana, por citar los más relevantes. En esta sintonía, (y con un cierto desfasaje temporal) la Argentina se inscribe en esta tendencia teniendo como referencias más directas las teorías bourdeanas y elisianas, la obra foucaultiana y los desarrollos teóricos posfoucaultianos.

El creciente intercambio entre intelectuales del campo académico de Brasil y Argentina, en particular a partir de los años 90, ha permitido, entre otras cosas, que las perspectivas teórico-metodológicas empleadas en ambos contextos tengan rasgos comunes, pero con usos y recepciones diferentes. Sin embargo, no existen hasta el momento trabajos que analicen ese movimiento teórico.
*Centro de Ciências da Educação, Universida-

de Federal de Santa Catarina.

${ }^{* *}$ Facultad de Humanidades y Ciencias de la Educación, Universidad Nacional de La Plata - Argentina. 


\section{Método}

En virtud de nuestro interés en la obra de Michel Foucault y de la carencia de estudios sobre la recepción de la obra de ese autor en el campo académico de la Educación Física, definimos el objetivo y el recorte del presente trabajo: analizar, en una perspectiva comparada entre Brasil y Argentina, los estudios más relevantes producidos en el campo académico de ambos países referenciados en la obra del pensador francés Michel Foucault, que han sido desarrollados por miembros del movimiento renovador de la Educación Física. Para definir los autores ha ser analizados usamos como criterios, por un lado, el rol de los conceptos foucaultianos para el desarrollo de la obra de los investigadores seleccionados, y, en segundo lugar (también de importancia) la relevancia que los autores tienen (o tuvieron) en el campo académico respectivo, brasileño o argentino. Para proceder al análisis identificamos cuáles herramientas teóricas foucaultianas eran utilizadas por cada autor e indagamos el grado de consistencia de esa apropiación. Solamente fueron seleccionados los textos de cada autor en los cuales categorías foucaultianas asumen un rol protagónico en la construcción del proceso argumentativo.

Para desarrollar este trabajo dividiremos el texto en tres partes: en la primera, expondremos brevemente cómo comprendemos los diferentes momentos de la obra foucaultiana. En la segunda, realizaremos un análisis de los modos de recepción de los conceptos en el campo académico de la Educación Física en Brasil y Argentina. En la parte final, que se divide en dos partes, presentamos primero una síntesis de los aspectos comunes y específicos de la recepción de la obra de Michel Foucault en Argentina y Brasil. Finalmente señalamos algunas posibilidades para pensar la educación y la Educación Física a partir del tercer dominio de la obra del pensador francés, que ha permanecido inexplorado en el campo de la Educación Física hasta el presente.

\section{Situando el horizonte de encuentro con el autor}

Es extenso el debate (en el que no entraremos en este momento) sobre la manera de sistematización de la obra de Michel Foucault, que va desde aquellos que sitúan su obra cronológicamente y postulan tres momentos: un momento arqueológico, un segundo momento de genealogía del poder y un tercer momento que tiene como objeto a la genealogía de la moral; pasando por otros de carácter temático, hasta aquellos que trabajan por "dominios" del sersaber, del ser-poder y del ser-consigo mismo ${ }^{3}$. Sin embargo, en este artículo trabajamos con la idea propuesta por un gran amigo de Foucault, el filósofo Gilles Deleuze. Para ese autor, en la obra foucaultiana podemos identificar tres dimensiones, que responden a las crisis que Foucault atravesó en su vida: la del saber, la del poder y la de la subjetivación ${ }^{4}$. Así, esas tres dimensiones "[...] constituyen una manera de vivir, una figura extraña en tres dimensiones, así como la mayor filosofía moderna [...]." ${ }^{\prime 4}$ (p.121). Esas tres dimensiones constituyen la tríada de la problematización de la subjetividad en Foucault.

Nuestro autor participa de este debate cuando hace un balance de su obra en el penúltimo curso que dictó en el Collège de France en 1983 y que lleva el nombre de "El gobierno de sí y de los otros". En ese momento FoucaulT ${ }^{5}$ nos dice que su obra tiene como objeto el "foco de la experiencia" que implica en la articulación de tres ejes que, a su vez, efectuaban un desplazamiento con relación a los modos de investigación vigentes hasta entonces. Son esos tres ejes y los desplazamientos respectivos:

Sustituir la historia de los conocimientos por el análisis histórico de las formas de veridicción; sustituir la historia de las dominaciones por el análisis histórico de los procedimientos de gubernamentalidad, y sustituir la teoría del sujeto o la historia de la subjetividad por el análisis histórico de la pragmática de sí y las formas adoptadas por ella: esas eran las tres vías de acceso mediante las cuales intenté circunscribir un poco la posibilidad de una historia de lo que podríamos llamar 'experiencias's (p.21-2).

Complementariamente a lo señalado, destacamos lo que el propio autor nos ofrece como una pista del eje que conduce su obra: la relación entre el sujeto y la verdad ${ }^{6}$. De la siguiente manera FoucaulT ${ }^{6}$ presenta ese eje:

intenté analizar de qué modo dominios como los de la locura, de la sexualidad, de la delincuencia pueden entrar en un cierto juego de la verdad y cómo, por otro lado, a través de esa inserción de la práctica humana, del comportamiento, en el juego de la verdad el propio sujeto es afectado (p.289).

La compleja (y hasta cierto punto infructífera) tarea de sistematizar un pensador esquivo a los rótulos, puede ser comprendida a partir de la propias palabras de Foucault, para quien escribir no es 
simplemente poner en juego la propia capacidad racional en relación a un objeto de conocimiento, sino que está relacionada con la experiencia de la propia transformación, con colocarse a sí mismo en riesgo. Para él: "cuando escribimos libros, deseamos que estos modifiquen enteramente todo aquello que pensábamos y que, al final, nos percibamos diferentes de lo que éramos en el punto de partida"6 (p.289).

Así, entendemos, con y a partir de Foucault que en su obra hay un eje conductor, pero, diferentemente de su auto-comprensión, consideramos que las maneras de aproximación a ese objeto difieren sensiblemente en sus otros momentos. Además, acordando con la idea de "dimensiones foucaultianas", entendemos que en las dos primeras dimensiones, saber y poder, la matriz epistemológica estructuralista de la cual se vale la obra foucaultiana, impide ver posibilidades que sean diferentes de la constitución de un sujeto-sujetado que aquellas realizadas; a pesar de que Foucault lo niegue, afirmando que donde hay poder siempre hay resistencia, porque el poder es siempre relacional y no substancial $^{6}$. En la obra foucaultiana como un todo, pero en particular en esas dimensiones la idea de cuerpo es la de una substancia material que es objeto de ejercicio del poder y que no escapa de los dispositivos de control y regulación que sobre él actúan. Las palabras de Francisco OrTEGA ${ }^{7}$ son muy oportunas:

Su obra [de Foucault] no proporciona una fenomenología del cuerpo adecuada para dar cuenta de su dimensión material, como es apuntada

\section{Resultados y discusión}

A continuación presentamos los modos de recepción del pensamiento de Michel Foucault en la producción académica de la Educación Física en Brasil y Argentina, a partir del análisis de artículos y libros de autoría de Homero Luis Alves de Lima, Alex Branco Fraga y Luiz Carlos Rigo para el caso brasileño y de, Ángela Aisenstein, Ricardo Crisorio y Pablo Ariel Scharagrodsky para el caso argentino.

\section{La recepción de Foucault en la Educación Física brasileña}

Los trabajos de corte foucaultiano en el campo académico brasileño de la Educación Física tienen, en su gran mayoría, como referencia las dos primeras dimensiones de la obra del filósofo francés, siendo la tercera dimensión un campo prácticamente por varios comentadores. La experiencia sensorial envuelta en el hecho de que tengamos un cuerpo no recibe la necesaria atención por Foucault, salvo el placer y el deseo, como únicas sensaciones que el cuerpo parece sentir. La experiencia vivida del cuerpo es eclipsada por el énfasis en los controles reguladores sobre él (p.198).

Ese cuadro cambió sensiblemente en la última dimensión, en la que emerge el protagonismo de una relación activa del sujeto para consigo mediada por la figura del maestro/guía y de la enunciación/ producción de la verdad de sí, y que resulta en la "metánoia", en la transformación de sí. En este momento la idea de "áskesis" es central, en la cual el decir verdadero es concebido como de ser del sujeto $^{8}$ y que consiste en " $[\ldots]$ un ejercicio sobre sí mismo a través del cual se procura elaborarse, transformarse y alcanzar un cierto modo de ser" 6 (p.265). En ese sentido, son elocuentes las palabras de Gilles Deleuze quien sugiere que Foucault precisaba establecer nuevas relaciones con el poder y el saber, que, a pesar de no estar motivadas por la fundación del sujeto o cualquier cosa semejante (idea que Foucault repelía), era motivada por la idea de encontrar líneas de fuga, espacios de resistencia ${ }^{4}$.

Por fin, destacamos que los conceptos foucaultianos que actuaron como herramientas teóricas para la construcción de los trabajos aquí analizados serán debatidos en el momento en el que discutamos las producciones seleccionadas. inexplorado. Se destacan los conceptos de saberpoder, de disciplina y de biopolítica como las principales "herramientas teóricas" que orientan los proyectos desarrollados. El movimiento de apropiación de la obra foucaultiana tuvo su inicio en los ańos 90 y se viene intensificando desde ese momento. En virtud de la extensión del texto, delimitaremos nuestro análisis del caso brasileño a tres autores que son exponentes destacados esa corriente teórica, Homero Luis Alves de Lima, Alex Branco Fraga y Luiz Carlos Rigo. Optamos por analizar esos autores porque son los que realizaron producciones referenciadas en la obra foucaultiana en diversos momentos de sus carreras académicas ${ }^{\mathrm{b}}$. Debemos destacar que esos autores se diferencian también con relación a su posicionamiento pedagógico, en cuanto Alex B. Fraga se sitúa en la corriente progresista de la 
Educación Física, Luiz C. Rigo y Homero L. A. de Lima, se filian a perspectivas pedagógicas pos-críticas.

El concepto de saber-poder orientó las reflexiones epistemológicas de Homero Luis Alves de Lima en finales de los ańos 90 del siglo XX. De ese autor seleccionamos dos artículos publicados en el año 2000. En esos trabajos el concepto de poder-saber es central para la construcción de las reflexiones del autor. Para Lima vivimos en un momento de crisis que tiene dos dimensiones, una crisis de los fundamentos y una crisis ética, siendo que ambas tienen como denominador común a la ciencia. Por un lado, no es posible más fundar la verdad en una concepción universal y neutra del conocimiento, porque la pretensión de saber total de la ciencia moderna muestra sus fisuras, y por otro lado, ese movimiento señala cómo la pretendida neutralidad científica no pasa de un mito. Por tanto es necesario reintroducir la dimensión ética en la discusión de la producción de conocimiento en la actualidad. Ese autor realizó una sistematización de la producción epistemológica de la Educación Física en dos grandes vertientes, una de matriz científica y otra de matriz pedagógica?. Lima entiende que la vertiente cientificista tiene serias limitaciones, porque pretende fundar una ciencia en un terreno que tiene como fundamento las dimensiones ética y estética. Por otro lado, a pesar de que Lima reconoce la mayor consistencia de la vertiente pedagógica en la construcción del objeto del área, él entiende que esa vertiente no puede (a pesar de quererlo) superar la concepción moderna de conocimiento pautada por la lógica disciplinar. Él también critica de esa vertiente el hecho de que su edificio conceptual tiene sus fundamentos en una idea universal de ser humano, que no sería posible a partir de una visión pos-moderna. Así, el autor entiende que la constitución de una teoría consistente para el campo de la Educación Física pasa por aceptar los desafíos de la agenda pos-moderna, que tiene en Foucault uno de sus representantes privilegiados.

Además, el autor hace, en otro momento ${ }^{10}$, una severa crítica de la pretensión totalitaria del pensamiento científico moderno, una racionalidad que se pretende única y universal, y que, por lo tanto, deslegitima las posibilidades de acceso a la verdad de otras racionalidades. Él también realiza una contundente crítica a la pretensión de neutralidad de la racionalidad científica a partir de Nieztsche y principalmente de Foucault, afirmando que la voluntad de saber está impregnada de voluntad de poder, por tanto el saber es siempre saber-poder. En ese sentido, entiende que, por el hecho de que la ciencia es el régimen de verdad por excelencia de la modernidad, la Educación Física buscó su legitimación a partir de un proyecto cientificista. Lima sostiene que es necesario afirmar la diversidad y el pluralismo de racionalidades, y estar abiertos para el juego de racionalidades situadas en un mismo nivel de validad que la pos-modernidad nos propone. En particular para el campo de la Educación Física el autor nos invita a pensar en un conocimiento no disciplinar o interdisciplinar, sino pos-disciplinar.

El emprendimiento teórico de Lima gana notoriedad en un momento en que posiciones aparentemente antagónicas, como la del positivismo y la del marxismo (ortodoxo) tenían una fuerte presencia en las discusiones epistemológicas de la Educación Física. Nos parece que en la medida que Lima asume como dada la condición de que todo saber conlleva una voluntad de poder y que la pretensión de validez universal sería una manifestación de ese carácter, su propuesta puede caer en el vaciamiento de la crítica inter-pares. El presupuesto de validar "a-priori" cualquier elaboración teórica puede hacer caer la propuesta del autor en la constitución de una comunidad de agentes que también se hacen inmunes a la crítica, una vez que se asume por válida toda y cualquier propuesta, sin que precise pasar por el ámbito de la discutibilidad pública.

Por fin, nosotros destacamos que Lima también fue uno de los precursores de la apertura de una agenda que hasta hoy no ha sido retomada en los estudios epistemológicos de la Educación Física brasilera: la dimensión estética o ético-estética como un horizonte posible para pensar la epistemología de esa área de conocimiento. El autor entiende, apoyado en Foucault, que "la crítica estética del cuerpo se revela como la actualidad de la Educación Física" (p.100). Después complementa diciendo: "[...] pensamos que la problemática de la legitimidad académica y social de la Educación Física podrá ser retomada de forma productiva por la vía de la discusión ética y estética del cuerpo." (p.101). Infelizmente ese problema no fue desarrollado posteriormente por el autor. Consideramos que la tercera dimensión de la obra foucaultiana que tiene como objeto a la subjetivación, contiene ricos elementos para elaborar ese problema, en particular por la centralidad que adquiere en esa dimensión la noción de "estética de la existencia".

A continuación discutiremos el trabajo de Alex Branco Fraga. De ese intelectual seleccionamos los dos libros de su autoría que han sido publicados como resultado de sus tesis de maestría y doctorado. 
En Fraga se destaca el uso de la categoría de biopolítica, pero también la noción de disciplina se hace presente. Su trabajo de maestría, intitulado "Corpo, identidade e bom-mocismo: cotidiano de uma adolescência bem-comportada", publicado en formato de libro en el año 2000, es un buen ejemplo del uso del concepto de disciplina. El trabajo retrata la investigación realizada por el autor en un colegio situado en el cinturón urbano de Porto Alegre - Rio Grande do Sul - Brasil. Fraga nos muestra como un orden discursivo marcado por una idea de "adolescencia normal", con una fuerte impronta del discurso moral del cristianismo católico que se impone en el cotidiano de la escuela constituyendo las identidades corporales de los niños y niñas que ahí se forman, resultando en cuerpos "bien educados".

A pesar de que el autor en diversos momentos afirme que su trabajo se sitúa en el punto de intersección de la intervención del poder sobre la vida, entre la disciplina y la biopolítica ${ }^{11}$, (p.101, 131 y ss.), en la obra no es posible observar la problematización de la dimensión biopolítica. A lo largo de todo el libro gana protagonismo la intervención sobre el cuerpo del individuo y no sobre el de la población, el cuerpo-especie.

A pesar de que el término disciplina no se observa frecuentemente en el texto, sí el concepto es un eje vertebrador del libro. Ilustramos esa afirmación en la forma como el autor concibe al espacio escolar, que revela la influencia de la categoría foucaultiana de disciplina. Leamos lo que nos dice:

Las clases de educación física en las escuelas, principalmente en las escuelas que poseen espacio reducido como el María Fausta, funcionan como el "tomar sol" en la prisión, donde los sujetos son autorizados a salir de sus cuadrículas, respirar en un ambiente más aireado y extender su mirada para algo más allá del límite de las cuatro paredes ${ }^{11}$ (p.118).

Asimilar el edificio escolar al de la prisión, con sus límites claros, con el cuadriculamiento del espacio y con el tiempo cronometrado, es un elemento que nos lleva a pensar más en la disciplina foucaultiana que en la noción de biopolítica.

En este momento es bueno recordar, aunque brevemente, el concepto de disciplina, que ha sido una herramienta teórica importante para los estudios sobre el cuerpo (principalmente en el ambiente escolar). Recordamos que Foucault ${ }^{12}$ concibe a la $^{2}$ disciplina como: "A estos métodos que permiten el control minucioso de las operaciones del cuerpo, que garantizan la sujeción constante de sus fuerzas y les imponen una relación de docilidad-utilidad, es a lo que se puede llamar 'disciplinas'” (p.159). Después agrega el autor: "La disciplina aumenta las fuerzas del cuerpo (en términos de utilidad económica) y disminuye esas mismas fuerzas (en términos de obediencia política)"12 (p.160). Así, en la obra foucaultiana la categoría de disciplina, cuñada en la primera mitad de los años 70 del siglo pasado, nos muestra el surgimiento de la intervención del poder político en el cuerpo del individuo, inaugurando una "anátomo-política" que se ocupa con los detalles, con el control minucioso y cotidiano de los gestos y las formas corporales, inscribiendo la norma en los cuerpos.

Finalmente, entendemos que la lectura de Fraga sobre el contexto escolar también se confronta con otro aspecto frecuente en los estudios escolares de corte foucaultiano, la crítica a la escuela como un espacio normalizador. Ese aspecto se torna más evidente cuando el autor presenta la manera como un niño experimenta el cambio de escuela. Ese cambio implica salir de un contexto (escuela de origen) en el que no había un orden normativo claro, para entrar en un espacio en el que la norma es claramente inscripta en los cuerpos (colegio María Fausta), y que marcan al "buen niño". Por fin el autor afirma que:

Escuela, familia e iglesia se articulan en líneas de fuerza y operan la conexión entre el sujeto y el discurso por medio de procedimientos 'quirúrgicos'. Algo que va limando los bordes, lapidando las asperezas, atravesando las más diferentes camadas del cuerpo hasta que se instale en él, hasta que parezca venir naturalmente desde dentro de él, hasta que, en fin, pase a ser irremediablemente su propio cuerpo ${ }^{11}$ (p.114).

En las palabras anteriores vemos un problema que gana gran importancia en el campo educacional. Si la escuela es una institución pedagógica, necesariamente debe tener un orden normativo a partir del cual conduzca la conducta de los educandos. Dicho de otra forma, en la lectura acusadora de la normalización que se opera en las instituciones educacionales se olvida que en ellas ese proceso es inherente a la función que socialmente le es atribuida. Si entendemos que la relación pedagógica por ser asimétrica es injusta y opresora; nos preguntamos si es posible una relación pedagógica horizontal en la cual los actores se sitúen en un mismo plano en las relaciones de poder. Nos parece que en esos análisis está como horizonte la expectativa de relaciones de poder puras, sin coerciones. Sabemos de las nefastas consecuencias que el discurso escolanovista (un tanto inspirado por ese ideal) produjo en la escuela. 
Foucault entendía que toda relación de poder implica dominación, o sea la pretensión de conducir la conducta del otro ${ }^{6}$, y que podemos aspirar a más o menos dominación, pero no a su inexistencia. Por otro lado, el filósofo francés pensaba que debemos tener otra relación con la norma, en la que nos constituyamos como sujetos éticos en la medida que, en una condición de libertad, podamos darnos la norma, siendo así "autonomos". Tal vez, podemos pensar con y a partir de Foucault (principalmente el Foucault de la dimensión de la subjetivación) en qué medida la escuela pueda ser un espacio en que vivamos otro tipo de relaciones de poder, con un mínimo de dominación o con una dominación "esclarecida y esclarecedora" y en el cual pueda haber lugar para la emergencia de subjetividades que vivan la experiencia de darse la norma.

En un momento posterior, en la realización de su tesis doctoral, Alex FraGA ${ }^{13}$ continúa su trabajo de investigación amparado en el referencial foucaultiano, pero esta vez teniendo como principal herramienta teórica al concepto de biopolítica. La tesis fue concluida en 2005 y publicada en formato de libro en 2006. El trabajo lleva el título de "Exercício da informação - governo dos corpos no mercado da vida ativa" y realiza una inteligente elaboración del concepto de biopolítica para acuñar un neologismo que el autor denomina "biopolítica informacional". Desde el punto de vista empírico, la investigación tuvo como foco los programas de promoción de la práctica de actividad física, enfatizando el programa "Agita São Paulo" y su correspondiente "maquinaria de la agitación"c, que fue concebido e implementado por el "Centro de Estudios del Laboratorio de Aptitud Física de San Cayetano del Sur" (CELAFISCS), dirigido por el Prof. Victor Matsudo, en cooperación con la Secretaría de Salud del Estado de San Pabloe.

La importante contribución de Fraga nos permite comprender de manera amplia y profunda las nuevas configuraciones discursivas de carácter informacional que inundan nuestra vida cotidiana a partir de los permanentes alertas (que se tornan imperativos) sobre la "obligación" (casi religiosa) de cumplir con nuestro deber de mantener un estilo de vida activo, que se justifica a partir de los riesgos a los cuales nos exponemos si no seguimos esos mandamientos y que se amparan en principios biomédicos resultantes de estudios de carácter epidemiológico ${ }^{13}$.

Para elaborar el concepto de biopolítica informacional, Fraga hace una lectura del mundo contemporáneo en el cual ve emerger la figura del mercado como una institución que pasa (al lado del Estado, y a veces sobreponiéndose a él) a regular la vida de la población. Por otro lado, el autor observa con propiedad que en la contemporaneidad el proceso de captura-control-cuidado de la vida de la población sufre un desplazamiento en la forma como se opera con la información relacionada a los parámetros para el control de vida del cuerpo-especie. En ese sentido, apunta que en la sociedad de control lo que interesa es hacer circular información (idea inspirada en el pensamiento de Gilles Deleuze) relacionada a lo que debe ser realizado para tener un "estilo de vida activo", en el contexto de una sociedad marcada por la "epidemia del sedentarismo", mal que aqueja a toda la humanidad. En ese sentido, destacamos la propiedad con la cual Fraga ${ }^{13}$ demuestra como los análisis estadísticos producidos por los "experts" del campo científico, en particular los que se dedican a la relación entre "actividad física y salud", sobre los procesos de morbi-mortalidad pasan a dar atención a la correlación entre sedentarismo y el desarrollo de enfermedades que minoran las fuerzas del cuerpo poblacional. Ese "ejercicio de la información" pasa a ser el mecanismo central de la lógica de la maquinaría biopolítica en la contemporaneidad.

Otro aspecto de debemos destacar en relación a la apropiación y reelaboración del concepto de biopolítica informacional se relaciona a los diferentes momentos en que se desarrolla la sociedad moderna. El autor llama "biopolítica analógica" a aquella forma de ejercicio del poder sobre la vida que se encontraba en las sociedades disciplinares, y que pasa a ser "informacional" en las sociedades de control ${ }^{13}$. En ese punto entendemos que es necesario hacer un breve análisis sobre esas relaciones conceptuales. En primer lugar no entendemos que sea posible (ni interesante) hacer una distinción entre sociedades disciplinares y sociedades de control, ya que el propio Foucault ${ }^{14}$ va a entender que la biopolítica, en cuanto tecnología de poder emergente en el siglo XVIII, a pesar de que haya sido iniciada posteriormente a las disciplinas, no la supera fundando una orden social, sino que: [...] esos dos mecanismos, uno disciplinario y el otro regularizador, no son del mismo nivel. Lo cual les permite, precisamente, no excluirse y poder articularse uno sobre el otro. Inclusive, podemos decir que, en la mayoría de los casos, los mecanismos disciplinarios de poder y los mecanismos regularizadores de poder, los primeros sobre el cuerpo y los segundos sobre la población, están articulados unos sobre otros (p.226).

Así, entendemos que para Foucault no hay una "superación" de la sociedad disciplinar por la irrupción de la tecnología de la biopolítica, sino una 
ampliación de la marca de la política moderna, el biopoder. De esa manera, no nos parece interesante situar como pares opuestos a la disciplina y la sociedad disciplinar por un lado; y a la biopolítica y la sociedad de control, por el otro. En ese sentido, es importante recordar que el propio pensador francés se ocupa en definir que "el poder sobre la vida", el biopoder, tiene una doble faz, una que opera sobre el cuerpo del individuo, la anatomopolítica del cuerpo humano, las disciplinas; y otra que opera sobre el cuerpo de la población, sobre el cuerpo-especie, la biopolítica de la especie humana ${ }^{15}$ (p.131-3). Resumiendo los argumentos anteriores, entendemos que la postura etapista con la que Fraga opera debería ser repensada, siendo el concepto de biopoder una adecuada herramienta teórica para ese emprendimiento.

Así, Fraga realiza, de manera coherente con los presupuestos teóricos que referencian su trabajo, un agudo diagnóstico del tiempo presente, revelando las estrategias de la biopolítica informacional en el mercado de la vida activa.

El tercer autor que analizaremos es Luiz Carlos Rigo. Serán objeto de análisis siete artículos que fueron producidos a lo largo de la primera década del siglo XXI y publicados en 2003, 2004, 2005, 2007, 2009 y 2012, siendo dos de ellos como único autor y cinco textos en co-autoría. El autor estudiado ha publicado más textos en el período que los aquí analizados, pero el recorte por los trabajos escogidos se fundamenta en dos criterios: 1) las publicaciones que tuvieron como principal referencia teórica, metodológica o teórico-metodológica a conceptos foucaultianos; y 2) los trabajos que fueron publicados en revistas relevantes del campo académico brasileño. Así tendremos una visión amplia de la manera de apropiación de los conceptos foucaultianos en los cuales Rigo referencia su producción académica. En el caso de Rigo la herramienta teórica foucaultiana más utilizada es el método de análisis del discurso.

Esos trabajos tienen como denominadores comunes al estudio del deporte, con énfasis para el fútbol (solamente un trabajo, el penúltimo publicado, no está relacionado al deporte) y al análisis del discurso referenciado en la obra de Michel Foucault. Otros conceptos foucaultianos también son objeto de apropiación, sin embargo solo son utilizados de manera tópica. En los textos que analizan el deporte se observan dos grandes líneas temáticas: por un lado, los estudios dedicados al análisis de agremiaciones, de prácticas y del ethos deportivo en el sur del Estado de Río Grande do Sul, por tanto con énfasis en procesos locales de constitución de subjetividades ${ }^{16-17}$; y por otro lado, el análisis de la producción del discurso mediático sobre la selección brasileña de fútbol. El texto que es dedicado a otra temática analiza la obesidad desde el punto de vista del marco legal que regula la identidad del obeso como figura jurídica. Algunos de los títulos de los artículos nos remiten a conceptos foucaultianos, a saber: "Memórias esportivas: uma história da subjetividade urbana"18; "Amizade, pertencimento e relaçóes de poder no futebol de bairro"17; "A nova 'Era Dunga': o treinador como um dispositivo"19.

Metodológicamente en todos los artículos, excepto el artículo sobre la memoria de cuerpos deportivizados ${ }^{20}$, se observa la referencia al análisis del discurso en perspectiva foucaultiana, siendo esta apropiada a partir de la mediación del análisis cartográfico propuesto por Suely Rolnik. Desde la perspectiva metodológica esa es la marca foucaultiana más importante en los trabajos de Rigo. Las principales obras de Foucault citadas son "El orden del discurso" y "La arqueología del saber", destacándose la primera. Sin embargo, notamos que el autor no se ocupó con detallar los procedimientos utilizados en la operacionalización del uso del método citado. Entendemos que esa postura se justifica porque el autor entiende que los caminos metodológicos deben ser ajustados al objeto investigado, lo que, sin dudas, es un argumento plausible. Esa postura se refleja en el pasaje que transcribimos a seguir y cuyo sentido es reproducido en todos los artículos que se utilizan de este recurso metodológico:

análisis del discurso en la perspectiva de Michel Foucault, según el cual el discurso es comprendido como prácticas que forman los objetos de que hablan, siendo constituidas por determinadas condiciones de posibilidades, políticas y epistemológicas (FOUCAULT, 1995; 1998). Junto al análisis del discurso, empleamos también algunos procedimientos y principios metodológicos del método cartográfico, según Rolnik (2006), sobre todo en la no obligatoriedad de delimitar, a priori, las fuentes que componen el corpus de la investigación, posibilitando cruzar y asociar fuentes de naturalezas distintas. Eso es porque la Cartografía permite inventar 'otros caminos nunca imaginados' (MAIRESSE, 2003, p.262) ${ }^{21}$ (p.544).

La idea de trabajar de manera abierta con la metodología citada es válida, pero también puede ser un arma de doble filo, porque puede hacer caer al autor en un uso poco riguroso de sus fuentes y a realizar afirmaciones generalizantes que ponen en riesgo las descubiertas del trabajo. Por otro lado, en la 
medida en que los principios teórico-metodológicos no tensionen (y sean tensionados) por el material empírico se puede caer, como es el caso en algunos de los trabajos aquí analizados ${ }^{16-17,19-20}$ en un privilegio de la dimensión descriptiva, en detrimento de la analítica y/o en un uso poco riguroso de los conceptos. Por ejemplo, en el artículo titulado "A nova 'Era Dunga': o treinador como um dipositivo" ${ }^{19}$ no es discutido en ningún momento el concepto de dispositivo, de capital importancia en la obra foucaultiana. Casos semejantes se observan en el empleo de otros conceptos altamente relevantes en el pensamiento de Michel Foucault como "relaciones de poder" ${ }^{17,20}$ o en la apropiación del concepto de infamia ${ }^{17,20}$. Solamente en dos artículos, cuyo objeto era el análisis de la producción discursiva mediática sobre la actuación de la "selección de Dunga" en la Copa América de 2007 17 y sobre el discurso de la derrota en la Copa del Mundo de Fútbol de $2006^{22}$ se puede identificar un uso consistente de los principios teórico-metodológicos y herramientas teóricas del análisis del discurso de corte foucaultiano, lo que cualifica sensiblemente al trabajo. En el primer texto citado leemos que, para Foucault, los discursos tienen un carácter performático, en la medida en que la realidad es producida por ellos, y, mejor, por la articulación de los diferentes discursos en una formación discursiva, y por las condiciones de posibilidad política y epistemológica de su producción ${ }^{22}$ (p.544). Los enunciadores son los protagonistas de la producción discursiva, y, por tanto de la constitución de la realidad en cuanto discurso. Con base en ese presupuesto, los autores entienden que:

La noción de que la Copa América podría representar un momento expresivo en la (re)construcción de un nuevo modelo de Selección Brasileña fue dada entre esas diversas formaciones discursivas (producidas por los diferentes enunciadores - los especialistas de la prensa deportiva), desplazándose en función de lo que los enunciadores apoyaban ${ }^{22}$ (p.547).

Ese procedimiento les permite a los autores explotar el potencial analítico del referencial foucaultiano del análisis del discurso.

Por otro lado, entendemos que los trabajos emprendidos por Rigo, en particular aquellos que se dedican al deporte local, han dado un importante aporte al campo de los estudios del deporte, por proponer una nueva mirada hacia las singularidades de los procesos y al protagonismo de los actores en la constitución de la subjetividad moderna, fuertemente relacionada al deporte, pero no a un deporte que puede ser tomado como dado universalmente, sino que se reproduce y recrea en las tácticas y estrategias del entramado de las relaciones intersubjetivas localizadas en un tiempo y en un lugar determinados.

\section{La recepción de Foucault en la Educación Física argentina}

La recepción de la obra foucaultiana en el campo de la Educación Física argentina, ha tomado variados y diversos matices, teniendo como principal campo de exploración problemas históricos de la Educación Física en el campo escolar. Tal como se ha producido para el caso brasileńo, en Argentina los trabajos de corte foucaultiano tienen, especial referencia a las dos primeras dimensiones de su obra, siendo la tercera dimensión un campo prácticamente inexplorado. En los trabajos que analizamos, encontramos como principales herramientas teóricas que se destacan las nociones de saber-poder, de disciplina y de biopolítica.

En esta línea hemos seleccionado, tres autores que para el caso argentino representan claros ejemplos de la recepción en el campo académico de la obra de Foucault, ellos son: Ángela Aisenstein, Ricardo Crisorio y Pablo Ariel Scharagrodsky. En primer término, analizaremos el trabajo de Ángela Aisenstein. Seleccionamos el texto titulado Cuerpo, escuela y pedagogia: Argentina, siglos XIX y XX, por su fuerte inscripción en los constructos teóricos que estamos discutiendo. La autora "intenta describir la genealogía de la asignatura escolar que hoy se conoce como Educación Física, en tanto componente del curriculum en los siglos XIX y XX"23 (p.22), siendo reconocido como uno de los componentes del "dispositivo escolar" para la "educación de los cuerpos". De este modo, la autora sostiene que la creación de la infancia y de la Pedagogía moderna, cuyo objetivo será el estudio de la educación, otorgan una instancia de limitación y normatividades respecto de la educación de los cuerpos.

Nos interesa señalar algunas de las preguntas que derivan del argumento. Entre ellas, si es la escuela un dispositivo? O si no sería posible pensar, en el marco de nuestra autora, en un dispositivo pedagógico y no necesariamente escolar?

Según dan cuenta los archivos, la educación del cuerpo forma parte del proceso de formación del ciudadano realizada por la institución escolar desde su constitución. El análisis se sitúa en los marcos de las normativas curriculares: "En los planes y programas para escuelas primarias diversas materias o ramos incluyen al cuerpo como tema o contenido"23 
(p.24). La autora muestra en los planes de 1877, 1884, 1887, 1901, 1908, 1910, 1914, 1916, 1921 y 1936 la presencia de las siguientes disciplinas, materias o ramos: higiene, gimnástica, ejercicios intuitivos (anatomía), ejercicios militares, ejercicios calisténicos, ejercicios y evoluciones militares, ejercicios y respiración al aire libre, articulación y puntuación, Educación Física, educación física y estética (Higiene), trabajo manual, entre otros.

En efecto, la creación de la Educación Física como disciplina centralizada en la educación del cuerpo y "del movimiento" no despoja al campo pedagógico de semejante responsabilidad. "La disciplinarización diferencial preanuncia la conformación de la Educación Física como asignatura escolar explícitamente diseñada para la educación de y por el cuerpo y el movimiento"23 (p.26). En consecuencia, según sus argumentos, la conformación de la Educación Física como asignatura escolar es un punto de inflexión y partida.

Una vez afianzada la Educación Física como componente curricular, comienza un denso proceso - que sigue vigente - en relación a la definición de los contenidos para la enseńanza. Claro está que la idea de normalización de los cuerpos - en pos de la mente- es sustentada por los grupos científicos y por el campo pedagógico, así como por la normalización de la formación de maestros y profesores y por la adopción de métodos y prácticas corporales (principalmente de orden gimnástico) provenientes del continente europeo.

De este modo, la necesidad de afianzar ejercicios útiles en sintonía con la higiene y la educación moral es ampliamente difundida y aceptada. A lo largo del siglo se registran indicaciones específicas respecto de cómo educar este cuerpo del ciudadano. Podríamos delinear en este sentido la vinculación con la hipótesis de Alex Fraga respecto de la constitución de los buenos modales ya señalada en este texto. Las palabras de AINSENSTEIN ${ }^{23}$ resumen bien los principios de ese proyecto:

En síntesis, una propuesta curricular centrada en la ejercitación analítica, sistemática, racional y metódica, con marcos de referencia fuertes y criterios de clasificación precisos (Bernstein, 1989), puede ser entendida bajo el concepto normalización o regulación heterónoma ${ }^{\mathrm{f}}$. Desde otra perspectiva, la decisión de enseñar a través del deporte y el juego en un programa variado y atrayente puede señalar otro modo de educar, de conformar y regular la personalidad de los alumnos, apelando a su natural impulso, autonomía y responsabilidad (p.42).

La matriz teórica que usará para describir estos procesos será el poder disciplinario - este poder que se ejerce sobre el cuerpo- que no alcanza para explicar el ejercicio del poder sobre la población. Sin embargo, nuestra autora ve emerger nuevas configuraciones, sublevaciones que reciben contraefectos de avanzada, nuevos refinamientos del ejercicio del poder. Sin embargo, la noción de poder disciplinar, le permite explicar ese poder que se ejerce -cuerpo a cuerpo-. Otra vez y tal como fue mencionado en el análisis del trabajo de Alex Fraga, se vislumbra la escuela como un espacio de normalización (afianzando el concepto en su negatividad).

No obstante, según la perspectiva foucaultiana, ya no se trata del poder disciplinar, del control cuerpo a cuerpo; se trata ahora - y hace más de un siglo- de un biopoder en escala populacional, de la biopolítica, que no excluye a la disciplina, sino que la complementa. De un poder que se ejerce sobre la vida de la población, sobre la producción, sobre el uso del tiempo, sobre modos específicos de formas de pensar que incluyen un cuidado de sí en tanto los nuevos modelos deseables, en función de nuevas o novedosas idealizaciones. El siguiente párrafo ilustra esta cuestión:

En la segunda mitad del siglo XX, los modos meticulosos y precisos de la sociedad disciplinaria comienzan a ser reemplazados por formas más liberadas de educación corporal en las escuelas. Ejemplo de esto es la amplia y expansiva adopción de los juegos deportivos de equipo en la enseñanza primaria y secundaria ${ }^{23}$ (p.42).

En consecuencia, el discurso podría traducirse de la siguiente manera: usted puede tener una vida de mejor calidad - y mayor duración- si se alimenta con productos naturales, si hace ejercicio, si va al médico y se controla. La idea de cuerpo que permanece es una idea ascética con la idea de auto-gerenciamiento, auto-control. Es lo que nuestra autora denomina biopolítica. Así, en la lectura de Ainsenstein, realizada a partir de un referencial foucaultiano, los conceptos de disciplina y biopolítica son centrales, y entiende que la Educación Física argentina, parte de una lógica centrada en el primero, para luego aproximarse del segundo. Sin embargo, vemos que, de forma análoga con el análisis de Alex Fraga, opera con esos conceptos de manera etapista.

En segundo lugar presentaremos a Ricardo Crisorio, quien representa otro aporte de referencia para el caso argentino. El texto que analizamos forma parte de un conjunto de publicaciones que el autor vincula a su tesis doctoral y en el que formula su propuesta de la Educación Corporal. En su texto Educación Física $^{24}$, sostiene que la noción de biopolítica es el soporte desde el cual se pueden explicar las prácticas 
de la Educación Física en la modernidad. El capítulo comienza con una afirmación que luego argumentará a lo largo del documento:

Su nacimiento (de la Educación Física) puede datarse con precisión. En la segunda mitad del siglo XIX los médicos y los fisiólogos promovieron y consumaron un movimiento de "reforma de la gimnástica” que procuró el desplazamiento de la gimnástica alemana por el sport inglés en toda la Europa continental. Esta intervención tuvo lugar en el marco de la medicina social urbana, organizada en el siglo XVIIIs, cuyo emergente y correlato científico fue la fisiología. Surgida del contacto de la profesión médica con otras ciencias afines, particularmente con la química, la fisiología cambió el concepto de naturaleza, que pasó a designar no solo el mundo exterior a la mente, como en Descartes, sino lo que debía considerarse normal respecto de los seres humanos ${ }^{24}$ (p.45).

Algunas consideraciones merecen nuestra especial atención. Por primera vez recuperamos en los estudios revisados una crítica al humanismo anclada en la crítica al Humanismo que realizara Michel Foucault en numerosos momentos de su obra. Así, se declara contrario a cualquier intento de entender a la Educación Física como parte de la continuidad del proyecto histórico occidental, que realizaría y potencializaría de forma plena la esencia de la humanidad. Además, en esas palabras Crisorio se posiciona a favor de una lectura de la Educación Física como una creación moderna, por tanto valiéndose del concepto foucaultiano de ruptura o corte histórico.

Entenderemos por humanismo a toda posición que se comprometa con una caracterización del sujeto humano entendido como invariante en los movimientos de la historia. Este sujeto invariante funcionaría además, como el sostén del discurso de la verdad en las ciencias humanas. En consecuencia, se afirmaría como núcleo de subjetividad universal, siendo entonces, condición de emergencia de la verdad y la objetividad (de lo intemporal e incondicionado) en el dominio siempre circunscrito históricamente y múltiplemente condicionado de lo social.

En este sentido, nos interesa señalar además las conexiones entre la crítica al humanismo- como crítica general a la modernidad-y las posibilidades explicativas que introduce Foucault, con la noción de biopolítica. Este movimiento foucaultiano se ve apropiado en la investigación que realiza Crisorio. De modo que, también enuncia una serie de antecedentes que dieron origen a este movimiento, mostrando que el real motivo de la reforma de la Gimnástica no fue la incorporación de los juegos, sino la adecuación a los nuevos desarrollos y requerimientos de la ciencia, especialmente de la fisiología.

La fisiología se ocupará de estudiar los modos para mejorar lo físico del hombre en tanto formas y movimientos. El estudio científico del movimiento humano se centrará a su vez en analizar aquellos movimientos que son naturales al hombre y aquellos que son artificiales. En este punto, podemos señalar que se observa el entendimiento foucaultiano de una ciencia que cosifica al hombre para estudiarlo y si se quiere, dominarlo ${ }^{\mathrm{h}}$. La creación de las ciencias humanas y la invención del hombre serán en este sentido, modos de favorecer su estudio y dominación.

Crisorio recupera la noción de trabajos de historia de Foucault, es decir, aquellos que hacen surgir el acontecimiento y la ruptura, y proveniencia en lugar de origen para argumentar que la Educación Física no sólo no se sitúa en continuidad con la gimnástica alemana, sino que además su trama histórica no puede explicarse solamente a través del dispositivo disciplinario. Éste no alcanza para explicarla sino a la vez con el dispositivo biopolítico de seguridad. Así, el autor enuncia su tesis proponiendo que es la población la que requiere de una Educación Física "capaz de poner el ejercicio al alcance de todos". Por otro lado, la noción de normalización y el papel de las ciencias humanas- con la invención del hombrecompletan el cuadro de este dispositivo. La siguiente cita recupera y explica claramente ésta cuestión: Claramente, la Educación Física no se sitúa en la continuidad de la "gimnástica" alemana (mucho menos de la griega) sino que se constituye contra ella.(...) la Educación Física se sitúa en un escenario y un tiempo que la proyección en el campo de la educación del dispositivo disciplinario no alcanza a explicar; al menos si no se le opone, y a la vez se lo completa, con el dispositivo biopolítico de seguridad. En la Europa de fines de siglo XIX la perspectiva biopolítica ya había modificado los dos ejes del proceso de disciplinarización: la noción de normalización y el papel de las ciencias humanas ${ }^{24}$ (p.51).

Es la población la que necesita una Educación Física para todos a fin de no dejar libradas a la gran mayoría "a todas las miserias físicas y morales que se derivan de la falta de ejercicio" ". Por otra parte, la posibilidad de modificar los dos ejes del proceso de disciplinarización refieren en primera instancia a que lo importante no es la normalización que deriva en "normales" o anormales", sino lo que toma 
otro lugar es la idea de norma. Foucault presenta la necesidad de plantear más una normación que una normalización. En una segunda instancia, el papel de las ciencias humanas en tanto responsables de lo "normal" y "anormal" durante toda la centuria.

Resumiendo, lejos de situar a la Educación Física en un espacio y tiempo lineal con la naturaleza humana, encontramos que Ricardo Crisorio defiende la tesis de situarla como un proyecto propio de la modernidad. Para hacerlo, enuncia una serie de acontecimientos que la desvincularán de la idea romántica- iluminista que la ilustra en el centro mismo de la antigüedad clásica. Sin embargo, esta noción de identificación tiene en su seno un sujetoque enunciado o silenciado- se inscribe en el concepto de normalización. Es por esto que el poder disciplinar -según nuestro autor- no alcanza para explicar la trama histórica de la educación física. Se nutrirá entonces del dispositivo biopolitico de seguridad para analizar la idea de los pares antagónicos: anormalnormal. Esta hipótesis abre un programa de trabajo que probablemente pueda ser explorado en otros escritos que aun no están publicados.

En tercer lugar mencionaremos el trabajo que viene realizando Pablo Ariel Scharagrodsky. Su trabajo se inscribe en un estudio de la educación del cuerpo a partir de los modos en que se ha sujetado a cierto orden corporal. Seleccionamos su texto "Los ejercicios militares en la escuela argentina: modelando cuerpos masculinos y patriotas a fines del siglo XIX", porque analiza como el cuerpo ha sido objeto y blanco de poder. El siguiente párrafo presenta el propósito de su trabajo:

El siguiente artículo pretende indagar en dicho orden (corporal) y sus efectos en la configuración y reconfiguración de los cuerpos masculinos centrándose en la disciplina escolar conocida como Educación Física. (...). En particular se analizará la gimnasia militar dirigida sólo a los varones, la cual ha sido una de las prácticas corporales relativamente hegemónica en las dos últimas décadas del siglo XIX y la primera década del siglo XX. Para dicho análisis se partirá desde una perspectiva teórica que, refiriéndose fundamentalmente a Michel Foucault, entre otros estudiosos, conceptualiza los procesos de escolarización, el dispositivo curricular y las disciplinas escolares como productores de sujetos, en este caso, de sujetos masculinos ${ }^{25}$ (p.106).

Para hacerlo, Scharagrodsky describe la matriz teórica desde donde analizar "el orden corporal" y sus efectos en la configuración y reconfiguración de estos cuerpos masculinos. En este caso, analiza las tecnologías del poder referenciándose en Michel Foucault. Siguiendo a nuestro autor, enuncia que ambas tecnologías - la disciplina y la biopolíticaforman parte de este proceso de sujeción del cuerpo en la modernidad. En este sentido, analiza la gimnasia militar destinada a varones como una forma de construcción de masculinidad en las dos últimas décadas del siglo XIX y en la primera década del siglo XX. Según el autor:

Después de la anatomía política del cuerpo, se instala una biopolítica de la especie humana, articulándose unos mecanismos sobre otros. Entre los objetivos de control de la biopolítica están la natalidad, la mortalidad y la longevidad. Esta nueva tecnología del poder trabaja sobre las poblacione $^{25}$ (p.109).

Los finales del siglo XIX y los comienzos del XX serán el escenario sobre el cual se montará un Estado que empieza a ocuparse de temas y problemas inherentes a la población. La Argentina de entonces, instaura un programa de educación que tiende a incorporar a sectores populares y marginados y a la diversidad de inmigrantes. Scharagrodsky señala la huella militar aun antes de la instauración de la Educación Física Escolar Argentina (Ley 1420 de Educación común, aprobada en 1884). La Educación Física se presenta en los Primeros Programas Escolares Argentinos como una educación que diferencia las prácticas corporales para varones y para mujeres. Los ejercicios militares para varones, tienden en este sentido a construir masculinidades. Es una gimnasia que enseńa a ser patriotas, disciplinados y varoniles.

El artículo avanza en la descripción de los ejercicios y de la pedagogía "del detalle" para enseñarlos. "La posición erecta y vertical es clave para este tipo de gimnasia. Esta posición debe ser acompañada de una determinada manera de caminar y dar pasos. (...) El caminar y estar de pié son expresiones de carácter"25 (p.118). La conformación de una Educación Física Escolar binaria, para varones y para mujeres, va a caracterizar el área curricular durante el siglo XX - y el XXI- en nuestro país. Sin embargo, la impronta de pedagogos que se oponen a estas gimnasias no tardará en llegar y diputará su legitimidad. En este sentido, nuestro historiador señala que ante los argumentos de pedagogos e higienistas de la época, la Educación Física militar se impondrá durante casi todo el siglo XX tomando al cuerpo como objeto y blanco de poder. Una anatomía política del detalle no bastará, según nuestro registro, para explicar la complejidad de fenómenos de los cuales el Estado tomará parte 
para la conformación de estos "nuevos ciudadanos". El apartado siguiente presenta algunas conclusiones y preguntas al respecto, como así también los resultados parciales de nuestra investigación.

\section{Consonancias y disonancias, alcances y límites de la recepción de la obra foucaultiana en Argentina y Brasil}

Se hace necesario en este momento del trabajo realizar un balance de los argumentos expuestos, focalizando los puntos en común y las divergencias entre los modos de recepción de la obra foucaultiana en el campo académico de la Educación Física de Argentina y Brasil, y avanzando en explorar el potencial analítico de las elaboraciones teóricas discutidas, así como también sus límites.

En primer lugar vale resaltar que en ambos países la recepción de la obra se ha realizado de manera tópica, es decir se ha aislado algún concepto foucaultiano (principalmente los conceptos de disciplina y biopolítica, pero también de discurso) y se lo ha utilizado como herramienta analítica para elucidar algún aspecto del ámbito de las prácticas pedagógicas de la Educación Física, sea en ambientes escolares o no escolares. Además, notamos que esa apropiación tópica de los conceptos ha sido realizada de forma "cristalizada", sin observar la policromía y el movimiento que el concepto sufrió a lo largo de la obra de Foucault. Además, debemos señalar que los tipos de estudios emprendidos en ambos países son diferentes; en Brasil predominan los estudios que se ocupan del presente, sea con problematizaciones de orden epistemológico, de análisis mediático o de técnicas y tecnologías de poder presentes en la Educación Física escolar y en programas de promoción de la práctica de actividad física. En Argentina prevalecen los trabajos tienen un corte historiográfico, o un interés histórico-filosófico.

Un segundo aspecto a resaltar, correlacionado a lo anterior, es la apropiación de conceptos situados en la "dimensión del poder" ", destacándose los conceptos de disciplina y biopolítica. Esa postura de los autores analizados en ambos países ha sido de gran valor para lanzar una luz crítica sobre el proceso de constitución del campo de la Educación Física, contribuyendo a desnaturalizar el desarrollo del mismo y a entender a la Educación Física como un terreno fértil para la implementación de las dos grandes tecnologías del poder, la anatomo- y la biopolitica, la intervención del poder sobre la vida del cuerpo-individuo, así como del cuerpo-especie.
Sin embargo, ese modo de trabajo en lo que contribuye a la crítica, limita también el avance en el sentido propositivo. En general, no se observa que los autores analizados tengan la intención de elaborar constructos de orden epistemológico y pedagógico de carácter propositivo, sino que tienen como objetivo utilizar esta matriz teórica para explicar problemas o acontecimientos que dan cuenta de la conformación del campo disciplinar. En ese sentido, consideramos que los análisis dejan poco espacio para proyectar posibilidades diferentes de aquellas que se realizaron, porque, en virtud del marco teórico escogido, no se observan los espacios o movimientos de resistencia a la forma en que las tecnologías del poder actuaron sobre el cuerpo individual y de la población, en el campo de la Educación Física. En consecuencia encontramos que en los trabajos analizados, se observa una relación lineal entre el cuerpo y el poder, siendo siempre de forma unidireccional del poder hacia el cuerpo.

En el interior de sus límites, consideramos muy valiosas las elaboraciones teóricas producidas en el campo de la Educación Física que se valieron de conceptos foucaultianas como las que analizamos. Sin embargo, entendemos que la obra foucaultiana nos brinda un conjunto de herramientas teóricas que hasta el momento han quedado inexploradas, en particular los conceptos propuestos por el pensador francés en su tercera dimensión. Si el movimiento de apropiación de ese legado no se ha realizado hasta la actualidad en la Educación Física, es probable que sea porque su publicación es de reciente data. En todo caso, consideramos que los conceptos de cuidado de sí, de "pharresía", de "ascesis", de estética de la existencia y de etopoiética, para citar los más relevantes, nos abren un horizonte promisor para el desarrollo del campo de la educación de forma general y de la educación física en particular.

En ese momento Foucault nos propone elaboraciones teóricas que buscan alejarse del determinismo estructuralista que predominaba en los períodos anteriores, en particular en la segunda dimensión, señalando para una nueva forma de constitución de la subjetividad, marcada por la implicación del sujeto con la verdad que enuncia, constituyéndose, así, en sujeto ético. En ese sentido Sílvio GALLO ${ }^{26}$ señala que la "ética del cuidado de sî" podría traer grandes contribuciones para (re)pensar la educación, pero que esa tarea ha sido deja en abierto por el campo educacional. La idea de verdad aquí presente nos permite un radical cuestionamiento de la relación con el saber hegemónica en la 
modernidad, que se pretende objetiva, universal, a-histórica y, principalmente, descomprometida con el sujeto que la formula y enuncia.

En este momento también se observa una inflexión (a pesar de no haber una posición unívoca en ese sentido) en la idea de cuerpo, el cuerpo deja de ser un lugar de mera pasividad en el que el poder se ejerce sin encontrar resistencias, para ser uno de los espacios en donde se constituye el sujeto ético. Además, vemos que en los diversos cursos constitutivos de la tercera dimensión el cuerpo pasa de ser un soporte para la constitución de la subjetividad para situarse en el lugar central en el que la subjetividad se manifiesta (aquí pensamos en el movimiento presente desde " $\mathrm{La}$ Hermenéutica del Sujeto" al "Coraje de la Verdad"), radicalizando la apuesta foucaultiana a favor de la finitud, de la frágil materialidad corporal y de la inmanencia. Por lo tanto, entendemos que la tercera dimensión foucaultiana es la que, desde el punto de vista pedagógico, contiene los elementos más fértiles para avanzar en la perspectiva propositiva. Por lo expuesto anteriormente entendemos que se nos presenta una agenda de investigación que pretendemos desarrollar.

Entendemos que la última dimensión foucaultiana nos presenta valiosas herramientas teóricas para cuestionar la tradición educacional, porque el problema de la verdad se sitúa en otro lugar. Ya no se coloca en el centro de la atenciones la relación entre un sujeto cognoscente y un objeto cognoscible, cuya aprehensión está dada por el acceso al conocimiento científico, sino que el foco está en la verdad de sí, a la cual solamente se tiene acceso por una relación ética de sí para consigo que tiene como mediador al "maestro". Ese aspecto nos remite a la relación pedagógica de la escuela moderna (o de la pedagogía moderna, que se hace presente en diversos ambientes educacionales, más allá de la escuela) y que presupone al profesor/a como un sujeto que media el desarrollo/apropiación/construcción/movilización de operaciones cognitivas, y que pasa a ser visto como un sujeto que orienta y conduce el proceso de creación ética de sí por parte del sujeto aprendiz, y que guarda con él una relación de compromiso e implicación. Si el cuidado de sí también implica el "hablar franco" ese acto de habla tiene que pasar también por las interdicciones del cuerpo y del alma, por un hablar del cuerpo como una dimensión constitutiva de sí. Por fin, vemos que la creación ética de sí, la estética de la existencia, tiene al cuerpo como uno de sus pilares, pero que debe actuar de forma articulada y complementaria con el cuidado del alma. Ese aspecto nos remite a las fronteras creadas en la modernidad y que escinden al sujeto y lo tratan en dos substancias "claras y distintas" que no participan de igual manera del ser del sujeto, ahora se trata de pensarlo más allá y más acá de esas fronteras, lo que implica pensar, por ejemplo, la adecuación de una asignatura llamada Educación Física y de pensar los procesos corporales de forma aislada y mecanizada.

En fin, aquí apuntamos apenas el inicio de intrigantes problematizaciones a partir de los conceptos acuñados por Foucault en su tercera dimensión. En todos ellos destacamos el potencial para lo posible, para lo por-venir, para el desplazamiento de las fronteras instituidas, en suma, pensamos que la Educación Física puede tener un promisor horizonte para su auto-creación a partir de una relación crítica de sí para consigo que se realice en el cuidado de sí, autocreación esa que tiene su fundamento en la eticidad.

\section{Notas}

a. El "movimiento renovador" de la educación física brasileña es entendido aquí como un guiño en el campo de la Educación Física que se realiza a partir de los años 80 del siglo XX, y que busca situar las bases para la legitimación y autonomización académica y pedagógica de la Educación Física. Proyecto llevado adelante desde diferentes perspectivas epistemológicas.

b. Vale mencionar también a la profesora Carmen Lúcia Soares, quien en su libro "Educação Física: raízes européias e Brasil”27, de gran impacto en el campo académico de la Educación Física brasileńa, se nutre de una matriz foucoltiana, en la que se incluyen autores brasileńos como Jurandir Freire Costa y Madel T. Luz. Sin embargo, no fue escogida porque en momentos posteriores abandonó al referencial foucoltiano, para privilegiar la lectura historiográfica del cuerpo propuesta por Georges Vigarello.

c. El concepto original en portugués es "maquinaria do agito". Con ese término el autor pretende representar la idea de mantenerse constantemente en movimiento, en estado de "agitación" permanente, tornada un imperativo.

d. Equivalente a un ministerio de salud provincial en Argentina.

e. Iniciativas de este género están siendo también implementadas en Argentina, siendo incentivadas por entidades con objetivos similares al CELAFISC, como por ejemplo la Red Argentina de Actividad Física (RAAF) (ver más información 
en http://www.rafargentina.com.ar/). Debe ser destacado que esa red tuvo como uno de sus promotores al Prof. Matsudo. Además, notamos el "Programa Nacional de Actividad Física para la salud", del que forma parte el proyecto "Argentina en Movimiento", que es tributario del "Agita São Paulo". Por fin, ese programa ha sido incluido más recientemente, con algunas modificaciones, en el "Plan Argentina Saludable" como una de sus tres líneas, denominada de "vida activa". (más información ver http://www.msal.gov.ar/argentina-saludable/index.html).

f. Las cursivas son de los autores.

g. El autor cita a pié de página: "Véase el artículo sobre Gimnástica en este mismo libro".

h. Véase "Las ciencias Humanas" en "Las Palabras y las cosas"28.

i. Crisorio cita a Lagrange F. La higiene del ejercicio en los niños y en los jóvenes. Madrid: Librería de José Jorro: 1894.

\begin{abstract}
Foucault's bodies: a genealogy of foucaultian's studies in the academic field of Physical Education in Brazil and Argentina
\end{abstract}

This paper aims to analyze the researche developed in the Physical Education academic field of two south American countries, Argentina and Brazil, based on Michel Foucault's work. Our analysis focuses three Brazilian researchers: Homero Luis Alves de Lima, Alex Branco Fraga and Luiz Carlos Rigo and three Argentinian ones: Ángela Aisenstein, Ricardo Crisorio and Pablo Ariel Scharagrodsky. We show how these investigations are mostly based on the second foucaultian dominium, as well their relevance to denounce conditions such as disciplinarization of the bodies and the bio political control of the population. Nevertheless, the 3rd dominium of Foucault's work is not referred. We assume that this dominium would be helpful to construct a pedagogical proposal for physical education, beyond mere criticism.

KEY woRDS: Michel Foucault; Academic field; Argentina and Brazil; Pedagogy of physical education.

\title{
Referencias
}

1. Bracht V. Educação física e ciências: cenas de um casamento (in)feliz. Ijuí, UNIJUÍ; 1999.

2. Medina JPS. A educaçáo física cuida de corpo... e mente. Campinas: Papirus; 1987.

3. Veiga-Neto A. Foucault e a educação. Belo Horizonte: Autêntica; 2011.

4. Deleuze, G. Conversaçôes. São Paulo: Editora 34; 1992.

5. Foucault M. El gobierno de sí y de los otros: curso en el Collège de France. Buenos Aires: Fondo de Cultura Económica; 2009.

6. Foucault M. Ditos e escritos V: ética, sexualidade, política. Rio de Janeiro: Forense Universitária; 2004.

7. Ortega F. O corpo incerto: corporeidade, tecnologias médicas e cultura contemporânea. Rio de Janeiro: Gramond; 2008.

8. Foucault M. La hermenéutica del sujeto: curso en el Collège de France (1981- 1982). Buenos Aires: Fondo de Cultura Económica; 2006.

9. Lima HA. Epistemologia, relativismo e educação física. Rev Bras Ciênc Esporte. 2000;22:65-77.

10. Lima HA. Pensamento epistemológico da educação física brasileira: controvérsias acerca do seu estatuto científico. Rev Bras Ciênc Esporte. 2000;21:95-102.

11. Fraga AB. Corpo, identidade e bom-mocismo: cotidiano de uma adolescência bem-comportada. Belo Horizonte: Autêntica; 2000.

12. Foucault M. Vigilar y castigar: nacimiento de la prisión. Buenos Aires: Siglo XXI; 2008.

13. Fraga AB. Exercício da informaçáo: governo dos corpos no mercado da vida ativa. Campinas: Autores Associados; 2006.

14. Foucault M. Defender la sociedad: curso en el Collège de France (1975-1976). Buenos Aires: Fondo de Cultura Económica; 2000.

15. Foucault M. História da sexualidade 1: a vontade de saber. Rio de Janeiro: Graal; 1985.

16. Rigo LC. Nomadismo e miscigenação no futebol pelotense. Movimento. 2003:9:149-61. 
17. Rigo LC. Amizade, pertencimento e relaçóes de poder no futebol de bairro. Pensar Prát. 2007;10:83-98.

18. Rigo LC, Pardo ER. Memórias esportivas: uma história da subjetividade urbana. Rev Bras Ciênc Esporte. 2004;25: 21-37.

19. Rigo LC, Freitas GS, Silva MR. A nova "Era Dunga": o treinador como um dispositivo. Motriz. 2012;18: 9-21.

20. Rigo LC, Pardo ER, Figueiredo MB, Rodrigues A, Silveira VT. Memórias de corpos esportivizados: a natação feminina e o futebol infame. Movimento. 2005;11:131-46.

21. Rigo LC, Freitas GS. Discursos de uma derrota: um estudo da produção discursiva sobre a eliminação da seleção brasileira na copa do mundo de 2006. Rev Bras Ciênc Esporte. 2009; :111-25.

22. Rigo LC, Freitas, GS, Pardo ER. A seleção brasileira de futebol na Copa América de 2007: uma demanda discursiva inacabada. Educ Fis/UEM. 2009;20:543-54.

23. Ainsentein A. Cuerpo, escuela y pedagogía: Argentina, siglos XIX y XX. In: Aisentein A, Scharagrodsky PA, compiladores. Tras las huellas de la educación física escolar Argentina: cuerpo, género y pedagogía - 1880-1950. Buenos Aires: Prometeo Libros; 2006. p.19-49.

24. Crisorio R. Educación física. In: Crisorio R, Giles M. Estudios críticos de educación física. La Plata: Ediciones Al Margen; 2009.

25. Schargrodksky PA. Los ejercicios militares en la escuela argentina: modelando cuerpos masculinos y patriotas a fines del siglo XIX. In: Aisentein A, Scharagrodsky PA, compiladores. Tras las huellas de la educación física escolar Argentina: cuerpo, género y pedagogía - 1880-1950. Buenos Aires: Prometeo Libros; 2006. p.105-34.

26. Gallo S. Repensar a educação: Foucault. Educ Soc. 2004;29:79-97.

27. Soares C. Educação física: raízes europeias e Brasil. Campinas: Autores Associados; 1994.

28. Foucault M. Las palabras y las cosas: una arqueología de las ciencias humanas. Buenos Aires: Siglo XXI; 2002.

Santiago Pich

Departamento de Estudos Especializados em Educação

Centro de Ciências da Educação

Universidade Federal de Santa Catarina

Caixa Postal 476 - Campus Trindade

88010-970 - Florianópolis - SC - BRASIL

e-mail: santiago.pich@ufsc.br

Recebido para publicação: 04/12/2012

1a. Revisão: 26/o6/2013

2a. Revisão: 19/12/2013

3a. Revisão: 28/03/2014

Aceito: 17/04/2014 